\title{
Bayern auf dem Weg zur Pflegekammer
}

\author{
In Sachen Berufsgesetz ist Österreich voraus
}

Während der Vorstoß der standespolitischen Organisationen in Österreich, eine Grundlage für die Einrichtung einer Pflegekammer zu schaffen, wieder einmal von den politisch Verantwortlichen abgewiesen wurde, ist das südlichste Bundesland unseres nordwestlichen Nachbarlands scheinbar einen Schritt weiter. Beim Berliner Kongress des Pflegemagazins Heilberufe - „Pflege 2011“ - wurde bekannt, dass Bayern als erstes Bundesland eine Pflegekammer einrichten will.

Die Deutschen Pflegeverbände kämpfen seit vielen Jahren für die Etablierung einer Pflegekammer - als Körperschaft des öffentlichen Rechts, denn nur so sind die Pflegekräfte den Berufsvertretungen von Apothekern und Ärzten gleichgestellt. Andreas Westerfellhaus, Präsident des Deutschen Pflegerats (DPR) e.V forderte denn auch alle anderen Landesregierungen auf, dem Beispiel Bayerns zu folgen. Seine Vision für das Jahr 2014: Der DPR hat sich in Bundespflegekammer umbenannt, Pflegende diagnostizieren, therapieren und evaluieren. Die gesetzlichen Grundlagen für eine Übernahme eigenverantwortlicher Tätigkeiten sind geschaffen und Pflegende verordnen auf Basis eigener Budgets. Pflege-Studiengänge sind flächendeckend eingeführt, die Ausbildung erfolgt nach internationalen Standards und das Bundesministerium für Gesundheit richtet eine Stabsstelle Pflege ein. Eine erste Etappe auf dem Weg dorthin stellen die Registrierung und das Engagement aller Pflegenden in den Verbänden dar sowie finanzielle Unterstützung und nicht zuletzt die fachliche Expertise.

„Wir wollen in diesem Jahr Großes für die Pflege erreichen“, stellte Annette Widmann-Mauz (CDU), Parlamentarische

\section{Pflegeblog}

Die Frage der Pflegekammer wurde nach dem vom Deutschen PROCARE Schwestermagazin Heilberufe veranstalteten Kongress in Berlin im Internet heftig weiterdiskutiert. Blog: http://blog.heilberufe-online.de/ unter Suche: Pflegekammer eingeben.

\section{Heilberufe Kongress}

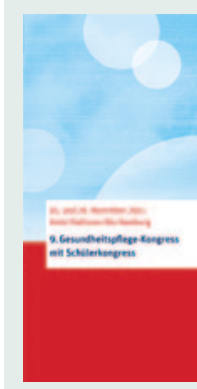

\section{Gesundheitspflege-Kongress}

Termin: 25. und 26. November 2011

Ort: Hamburg

Informationen: Urban \& Vogel $\mathrm{GmbH}$,

Kongressorganisation, Projektleiterin: Andrea Tauchert,

Heidelberger Platz 3, D-14197 Berlin

Deutschland

Telefon: 0049/30/82787 - 5510

Telefax: 0049/30/82787 - 5511

E-Mail: andrea.tauchert@springer.com

Staatssekretärin beim Deutschen Bundesminister für Gesundheit, fest. Der Pflegebedürftigkeitsbegriff soll überarbeitet, die Angebotspalette der Pflegeversicherung weiter ausgebaut werden.

In der Schaffung eines Berufsgesetzes ist allerdings Österreich den Deutschen Nachbarn voraus. Dieses steht noch auf der Liste der Vorhaben. „Die drei Pflegefachberufe werden wir noch in dieser Legislaturperiode mit einem neuen Berufsgesetz zusammenführen", sagte die Staatssekretärin. Der DPR fordert jedenfalls umfassende Inhalte in einem derartigen Berufsgesetz. Das aktuelle Eckpunktepapier des DPR verlangt die Ergänzung der Ausbildungsreform um die Festlegung prioritär vorbehaltener Aufgaben der Pflege. „Nur eine gesetzliche Regelung, wer nach der Ausbildung was mit welcher Qualifikation tun darf, ermöglicht", so Westerfellhaus, „Rechtssicherheit und eine Sicherstellung der Versorgungsstrukturen." Und nur mit einer Ausgestaltung der Aufgaben und Kompetenzen der professionell Pflegenden könne die Eigenständigkeit der Pflegeberufe weiterentwickelt und damit der Beruf attraktiver werden.

\section{Übertragung von Aufgaben}

Die Frage: „Delegation oder Substitution?“ wird derzeit in Deutschland heftig diskutiert und sollte ebenfalls durch ein Berufsgesetz geklärt werden. Laut Pflegeweiterentwicklungsgesetz von 2008 können Krankenkassen Modellprojekte auflegen, in denen Pflegekräften weit reichende Aufgaben zuerkannt werden. Pflegepersonal soll Verbands- und Pflegehilfsmittel verordnen und Aufgaben übernehmen, die im Bereich der Heilkunde liegen. Der Gemeinsame Bundesausschuss (G-BA) soll dazu eine Richtlinie erarbeiten, doch auch nach zwei Jahren hat das Gremium noch nichts vorlegen können. „Wir kommen zu keiner Entscheidung, weil wir bisher keinen Konsens erreicht haben", bedauerte Dr. Rainer Hess, Vorsitzender des G-BA. Es handele sich zwar eigentlich nur um eine Legalisierung eines Prozesses, der sich in der Praxis schon längst abspiele, dennoch: „Die Ärzte befürchten eine Qualitätsminderung.“ Ärztevertreter könnten sich zwar eine Delegation vorstellen, als Übertragung der Durchführungsverantwortung, nicht aber eine Substitution, als Übertragung der Entscheidungskompetenz.

Auch Widmann-Mauz sprach sich für eine Delegation aus - „Das hat sich bewährt" - und äußerte sich bei der Substitution zurückhaltend, weil das „mit nicht unerheblichen Risiken aus haftungsrechtlicher Sicht" verbunden sei. So oder so, eine Voraussetzung müsse immer gegeben sein: „Die Ärztin oder der Arzt muss in jedem Fall die Diagnose festlegen“, stellte Hess klar.

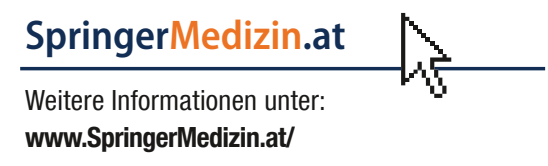

